\title{
Detection of Nasopharyngeal Carcinoma by MR Imaging: Diagnostic Accuracy of MRI Compared with Endoscopy and Endoscopic Biopsy Based on Long-Term Follow-Up
}

\author{
A.D. King, A.C. Vlantis, T.W.C. Yuen, DB.K.H. Law, K.S. Bhatia, B.C.Y. Zee, J.K.S. Woo, A.T.C. Chan, K.C.A. Chan, and A.T. Ahuja
}

\begin{abstract}
BACKGROUND AND PURPOSE: Our previous nasopharyngeal carcinoma detection study, comparing MR imaging, endoscopy, and endoscopic biopsy, showed that MR imaging is a highly sensitive test that identifies nasopharyngeal carcinomas missed by endoscopy. However, at the close of that study, patients without biopsy-proved nasopharyngeal carcinoma nevertheless had shown suspicious abnormalities on endoscopy and/or MR imaging. The aim of this study was to determine whether there were any patients with undiagnosed nasopharyngeal carcinoma by obtaining long-term follow-up and to use these data to re-evaluate the diagnostic performance of MR imaging.
\end{abstract}

MATERIALS AND METHODS: In the previous study, 246 patients referred to a hospital ear, nose, and throat clinic with suspected nasopharyngeal carcinoma, based on a wide range of clinical indications, had undergone MR imaging, endoscopy, and endoscopic biopsy, and 77 had biopsy-proved nasopharyngeal carcinoma. One hundred twenty-six of 169 patients without biopsy-proved nasopharyngeal carcinoma underwent re-examination of the nasopharynx after a minimum of 3 years, including 17 patients in whom a previous examination (MR imaging = 11; endoscopy = 7) had been positive for nasopharyngeal carcinoma, but the biopsy had been negative for it. Patients with nasopharyngeal carcinoma were identified by biopsy obtained in the previous and this follow-up study; patients without nasopharyngeal carcinoma were identified by the absence of a tumor on re-examination of the nasopharynx. The sensitivity and specificity of the previous investigations were updated and compared by using the Fisher exact test.

RESULTS: One patient with a previous positive MR imaging finding was subsequently proved to have nasopharyngeal carcinoma. Nasopharyngeal carcinomas were not found in the remaining 125 patients at follow-up, and the previous positive findings for nasopharyngeal carcinoma on MR imaging and endoscopy were attributed to benign lymphoid hyperplasia. The diagnostic performances for the previous MR imaging, endoscopy, and endoscopic biopsy were 100\%, 88\%, and 94\%, respectively, for sensitivity, and $92 \%$, $94 \%$, and $100 \%$, respectively, for specificity; the differences between MR imaging and endoscopy were significant for sensitivity $(P=.003)$ but not specificity $(P=$ .617).

CONCLUSIONS: MR imaging detected the $12 \%$ of nasopharyngeal carcinomas that were endoscopically invisible, including 1 cancer that remained endoscopically occult for several years. Lymphoid hyperplasia reduced the specificity of MR imaging.

ABBREVIATION: NPC = nasopharyngeal carcinoma

$\mathrm{N}$ asopharyngeal carcinoma (NPC) is a radiosensitive tumor that can often be cured when detected early, but the naso-

Received February 9, 2015; accepted after revision May 2.

From the Department of Imaging and Interventional Radiology (A.D.K., T.W.C.Y., B.K.H.L., K.S.B., A.T.A.); Department of Otorhinolaryngology, Head and Neck Surgery (A.C.V., J.K.S.W.); Jockey Club School of Public Health and Primary Care (B.C.Y.Z.); and Departments of Clinical Oncology (A.T.C.C.) and Chemical Pathology (K.C.A.C.), The Chinese University of Hong Kong, Prince of Wales Hospital, Hong Kong S.A.R., China.

This work was supported by a grant from the Research Grants Council of the Hong Kong Special Administrative Region, China (Project No. CUHK4656/12 and SEG_CUHK02).

Please address correspondence to A.D. King, MD, FRCR, Department of Imaging and Interventional Radiology, Faculty of Medicine, The Chinese University of Hong pharynx is a clinically silent region and patients often present in the later stages of the disease. ${ }^{1}$ Currently, the investigations for confirmation of NPC entail a nasopharyngeal endoscopy followed by an endoscopically directed biopsy at the site of an abnormality or sampling biopsies from an endoscopically normal nasopharynx. These methods may miss small nasopharyngeal carcinomas, however, because they are typically submucosal tumors or tumors located at the lateral aspect of the pharyngeal

Kong, Prince of Wales Hospital, 30-32 Ngan Shing St, Shatin, New Territories, Hong Kong S.A.R., China; e-mail king2015@cuhk.edu.hk

- - Indicates open access to non-subscribers at www.ajnr.org

http://dx.doi.org/10.3174/ajnr.A4456 
MRI Grade

\author{
Grade $1=$ normal \\ Grade 2 = low index of \\ suspicion of NPC
}

Grade 3 = high index of

suspicion of NPC

Grade $4=$ NPC

\section{MRI Appearance}

Symmetric mucosa $<3 \mathrm{~mm}$ thick with or without small retention cysts

1) Symmetric mucosal thickening $>3 \mathrm{~mm}$ thick (marked contrast enhancement $>\mathrm{NPC}$ ) with or without small retention cysts or a Thornwaldt cyst, or 2) symmetric enlargement of the adenoid in the central roof/upper posterior wall with a striped appearance \pm a smooth band (mild contrast enhancement $<$ NPC) extending from the roof down the posterior and lateral nasopharyngeal walls

Asymmetric: asymmetry between the right and left sides of the nasopharynx in grade 1 or 2 appearance

Asymmetric mucosal thickening of homogeneous intermediate signal intensity on T2-weighted images and moderate contrast enhancement on T1-weighted images (performed without fat saturation), with or without infiltration outside the nasopharynx, or a focal homogeneous enhancing mass with or without infiltration outside the nasopharynx

${ }^{a}$ Grades 1 and 2 were negative for NPC; grades 3 and 4 were positive for NPC.

recess. These small nasopharyngeal carcinomas are becoming an even greater diagnostic challenge in the era of NPC screening ${ }^{2}$ by using the Epstein-Barr virus as a surrogate, whether by serology, DNA, or nasopharyngeal brushings.

New methods for the early detection of NPC, such as narrow band imaging ${ }^{3,4}$ and sonography, are currently undergoing evaluation, ${ }^{5}$ but one of the most promising modalities in this regard is MR imaging. MR imaging has been used to stage biopsy-proved NPC for nearly 20 years, ${ }^{6-8}$ but it is also ideally suited for the initial detection of the primary tumor. ${ }^{9}$ In a previous prospective NPCdetection study, ${ }^{10}$ we compared the diagnostic accuracy of nasopharyngeal MR imaging with that of nasopharyngeal endoscopy and endoscopic biopsy. The results of that study showed that MR imaging is a highly sensitive technique for NPC detection and one that has a significantly higher sensitivity for NPC detection than endoscopy. At the close of that study, however, there were subjects who nevertheless had shown MR imaging or endoscopic abnormalities that were suspicious for NPC, but the biopsy had been negative for NPC. Therefore, we planned to determine whether there were any patients with undiagnosed NPC, by obtaining long-term follow-up of all those patients without biopsy-proved NPC, on the basis of re-examination of the nasopharynx after a minimum of 3 years. Our goal was to determine whether the previous MR imaging examinations had been able to identify any further nasopharyngeal carcinomas or indeed whether the previous MR imaging examinations had missed any nasopharyngeal carcinomas. In addition, we planned to evaluate the MR imaging examinations with false-positive findings to determine whether the specificity of MR imaging could be improved.

\section{MATERIALS AND METHODS \\ Previous Study}

Patients with suspected NPC had been entered into the previous prospective study ${ }^{10}$ comparing MR imaging, endoscopy, and endoscopic biopsy (biopsy from the site of an endoscopic abnormality or sampling biopsies from the endoscopically normal nasopharynx). Full details have been published previously, ${ }^{10}$ but to summarize, patients with suspected NPC were recruited from the ear, nose, and throat out patient clinic at a referral hospital in a region where NPC is endemic. To avoid bias, we based a clinical suspicion of NPC on a wide range of indications, such as positive serology for Epstein-Barr; metastatic cervical lymph nodes; ${ }^{18} \mathrm{~F}$ fluorodeoxyglucose positron-emission tomography scan with abnormal findings; and nonspecific symptoms (such as epistaxis, blood-stained saliva, nasal obstruction, or hearing loss) in the presence of a nasopharyngeal abnormality on flexible nasopharyngeal endoscopy.

MR imaging targeted to the nasopharynx had been obtained in all patients by using the following 4 sequences: 1) axial fat-suppressed T2-weighted images; 2) axial T1-weighted spin-echo images; 3 and 4) T1-weighted spin-echo images after a bolus injection of contrast in the axial (3) and coronal (4) planes. MR imaging had been graded independently by 2 radiologists (A.D.K and K.S.B with 15 and 4 years' experience, respectively, in head and neck radiology), and in cases of discordance, the grade had been obtained by consensus. MR imaging had been assessed without knowledge of the endoscopic findings and vice versa. MR imaging findings had been designated as NPC-negative (grades 1 and 2) or NPC-positive (grades 3 and 4), and details of the MR imaging grading system are shown in Table 1. A diagnosis of NPC had been made by histology from a nasopharyngeal biopsy, which had been obtained from either the initial endoscopic biopsy (directed by the endoscopic examination or sampling biopsies from an endoscopically normal nasopharynx) or a repeat biopsy directed by the MR imaging examination. The previous study had performed MR imaging, endoscopy, and endoscopic biopsy in 246 patients; at the close of that study, 77 patients had biopsyproved NPC and 169 patients did not. ${ }^{10}$

\section{Long-Term Follow-Up Study}

The current study aimed to follow up those 169 patients without biopsy-proved NPC in the previous study at a minimum of 3 years by using MR imaging and endoscopy to re-examine the nasopharynx. The presumption was that any small undiagnosed NPCs from the previous study should have grown and would now be more apparent and amenable to biopsy; therefore, biopsy was performed only in those patients with a suspected tumor on re-examination.

The previous study and follow-up study were approved by the institutional review board with written informed consent obtained.

\section{Statistical Analysis}

Patients with NPC were identified on the basis of a biopsy-proved NPC obtained in the previous and this follow-up study. Patients without NPC were identified by the absence of a biopsy-proved NPC after re-examination of the nasopharynx in this follow-up study. The sensitivity, specificity, negative predictive value, posi-

AJNR Am J Neuroradiol 36:2380-85 Dec 2015 www.ajnr.org

2381 


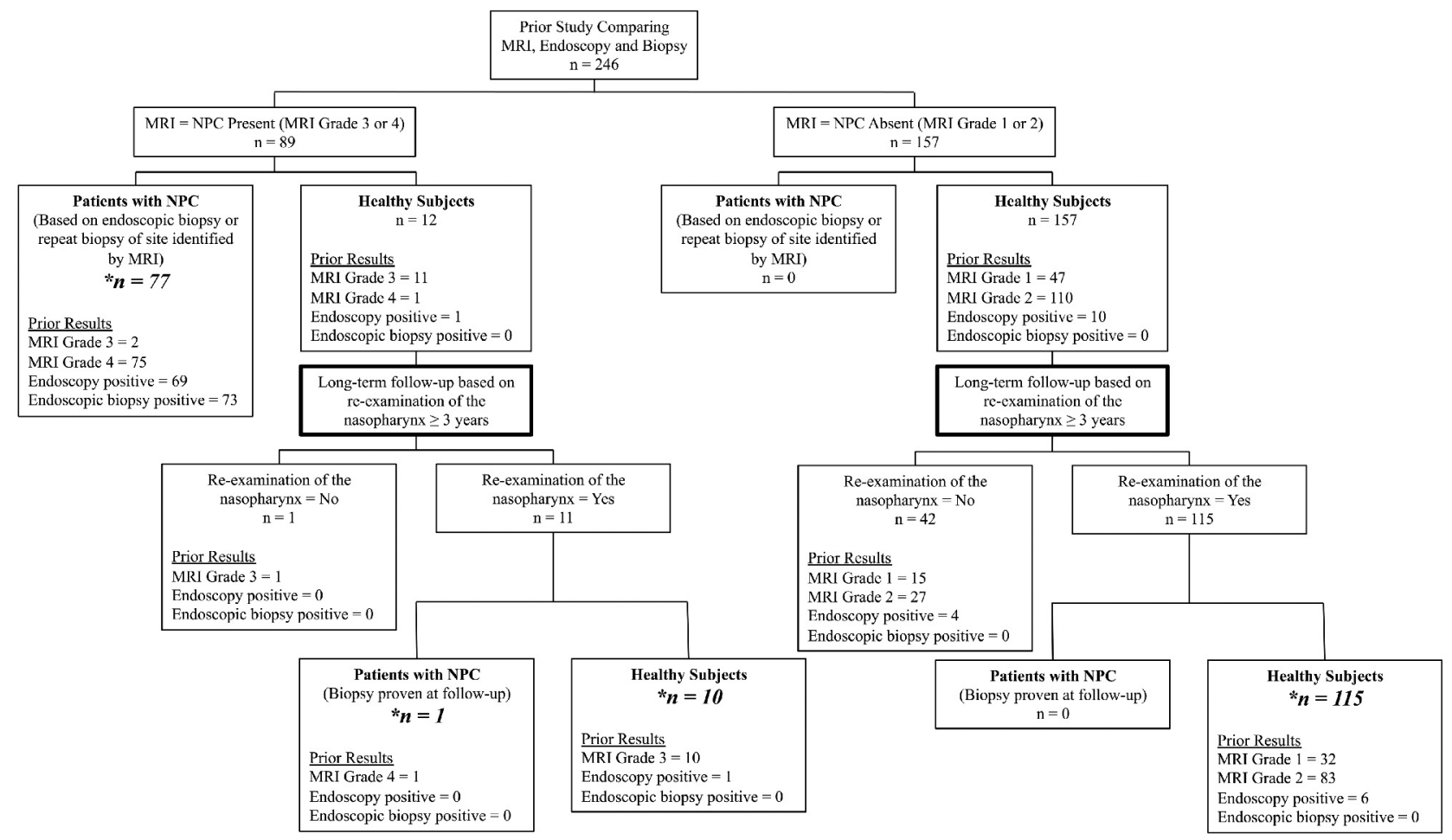

FIG 1. Flow chart of the study design.

tive predictive value, and the accuracy of the previous nasopharyngeal MR imaging, endoscopy, and endoscopic biopsy were updated by using the long-term follow-up data. The sensitivity and specificity were compared by using the Fisher exact test. A $P$ value $<.05$ was considered statistically significant.

\section{RESULTS}

\section{Long-Term Outcome}

Forty-three of 169 (25.4\%) patients without any clinical history of NPC were excluded from analysis because they did not undergo re-examination of the nasopharynx (Fig 1). They comprised 35 patients with follow-up of $>3$ years (mean, 62.3 months; range, 39-86 months), of whom 31 declined further nasopharyngeal examination, 3 died from causes unrelated to NPC, and 1 could not be contacted; and 8 patients with follow-up of $<3$ years (mean, 11.2 months; range, 0.5-32 months), of whom 7 died from causes unrelated to NPC and 1 could not be contacted. Of the 43 patients excluded from analysis, 5 had previous positive examination findings (MR imaging, $n=1$; endoscopy, $n=4$ ); 1 with a previous positive endoscopy examination finding had follow-up for 19 months, while the other 4 patients had follow-up ranging from 39 to 73 months (mean, 56 months).

One hundred twenty-six of 169 patients $(74.6 \%)$ had re-examination of the nasopharynx and were included in the analysis (Fig 1). Re-examination was performed after 3 years (mean, 58.5 months; range, 36-84 months) comprising MR imaging and endoscopy $(n=113)$, MR imaging only $(n=4)$, and endoscopy only $(n=9)$. NPC was identified in $1 / 126$ patients, a patient with a previous positive grade $4 \mathrm{MR}$ imaging finding but without a diagnosis of NPC at the close of the previous study. MR imaging had identified a tumor in the pharyngeal recess, but endoscopy had been negative for tumor and endoscopic biopsies had not revealed a tumor even after the repeat biopsy at the site of the abnormal MR imaging findings. This small tumor grew very slowly until the NPC was confirmed by endoscopy and histology 43 months later (Fig 2). NPC was not found in the 10 patients whose previous MR imaging findings had been positive on the basis of asymmetry in a generalized lymphoid hyperplasia pattern (grade 3) or in the 115 patients whose previous MR imaging findings had been negative (grade $1=32$; grade $2=83$ ). NPC was not found in the 7 patients whose previous endoscopic findings had been positive.

\section{Updated Results of the Previous NPC Detection Study Based on Long-Term Outcome}

The updated diagnostic performances of MR imaging, endoscopy, and endoscopic biopsy are shown in Table 2 and are based on data from 203 patients, comprising 78 patients with biopsyproved NPC (77 detected during the previous study and 1 during follow-up) and 125 patients without NPC based on re-examination of the nasopharynx after a minimum of 3 years (Fig 1).

NPC was present in 76/76 patients with a positive MR imaging finding showing a tumor (grade 4); 9 of these NPCs could not be visualized by endoscopic examination and 7 of these 9 NPCs had involved the pharyngeal recess on MR imaging. NPC was present in 2/12 patients with a positive MR imaging finding based on asymmetry in a generalized lymphoid hyperplasia pattern (grade 3 ); tumors in the nasopharyngeal wall of both patients were identified by endoscopy and biopsy. None of the remaining 10 patients with a positive grade 3 MR imaging finding had NPC: 9 with a negative endoscopy examination finding and 1 with a positive endoscopy examination finding in the nasopharyngeal wall (whose abnormal findings on MR imaging and endoscopy had regressed at 61 months). Analysis of these 10 grade 3 (Fig 3 ) false- 

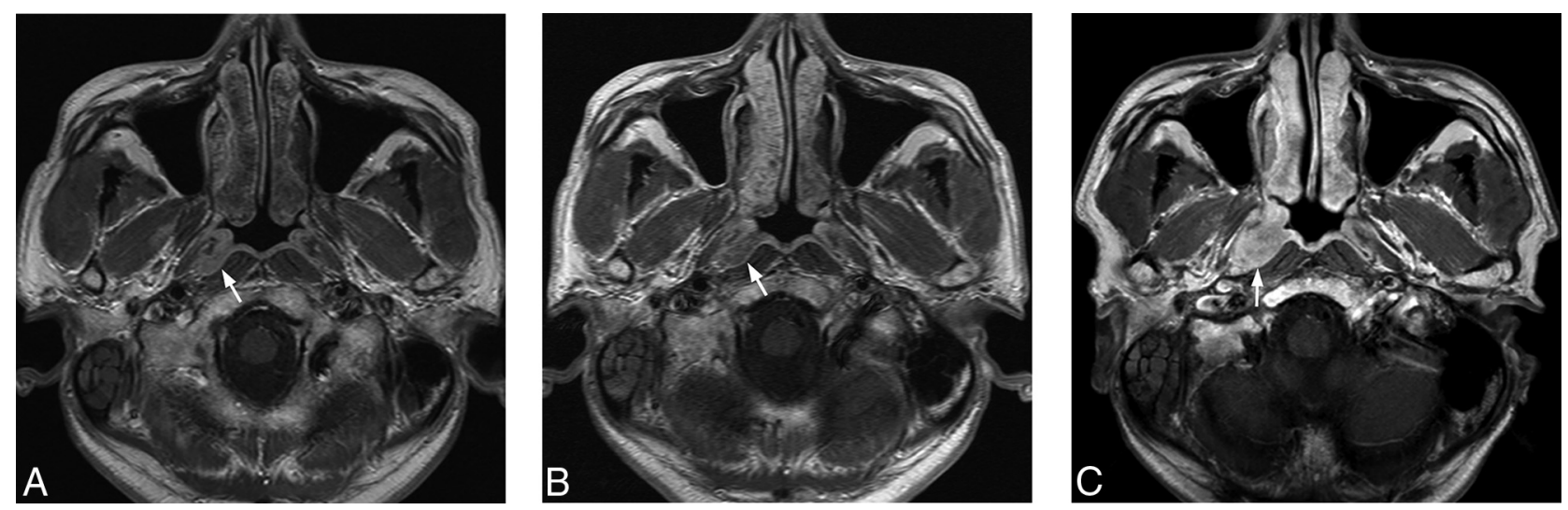

FIG 2. Axial T1-weighted postcontrast MR imaging of a 48-year-old man with NPC (arrow). A, Note a small moderately contrast-enhancing NPC in the right pharyngeal recess on MR imaging at presentation (grade 4), which was not detected by endoscopy or endoscopic biopsy or at repeat biopsy targeted to the site of the MR imaging abnormality. B, Persistent NPC on MR imaging is seen at 31 months, but without a tumor on endoscopic examination. A further biopsy was declined. C, An increase in the size of the NPC on MR imaging at 43 months when the tumor was confirmed by endoscopy and biopsy.

Table 2: NPC detection-grading of the previous MRI and updated diagnostic performances of the previous MRI, endoscopy, and endoscopic biopsy based on long-term outcome at 3 years in 203 patients (78 with NPC and 125 without NPC)

\begin{tabular}{|c|c|c|c|}
\hline & MRI & Endoscopy & Endoscopic Biopsy $^{\mathrm{a}}$ \\
\hline True-Positive & 78 & 69 & 73 \\
\hline & Grade $3(n=2)$; grade $4(n=76)$ & & \\
\hline True-Negative & Grade $1(n=32)$; grade $2(n=83)$ & 118 & 125 \\
\hline False-Positive & $\begin{array}{c}10 \\
\text { Grade } 3(n=10) ; \text { grade } 4(n=0)\end{array}$ & 7 & 0 \\
\hline False-Negative & Grade $1(n=0) ;$ grade $2(n=0)$ & 9 & 5 \\
\hline Sensitivity (\%) & 100 & 88 & 94 \\
\hline Specificity (\%) & 92 & 94 & 100 \\
\hline PPV (\%) & 89 & 91 & 100 \\
\hline NPV (\%) & 100 & 93 & 96 \\
\hline Accuracy (\%) & 95 & 92 & 98 \\
\hline
\end{tabular}

Note:-PPV indicates positive predictive value; NPV, negative predictive value.

a Endoscopic biopsy is endoscopically directed biopsy obtained at the site of the abnormality seen at endoscopy or sampling biopsies obtained from the endoscopically normal nasopharynx.

positive MR imaging examination findings had shown a benign pattern at the adenoid, which extended into the adjacent nasopharyngeal walls but with minor asymmetry between the left and right sides of the nasopharynx, which involved the adenoid $(n=$ $2)$, nasopharyngeal walls $(n=5)$, or both the adenoid and the walls $(n=3)$. These false-positive MR imaging examination findings have been attributed to asymmetric benign lymphoid hyperplasia. Six of 7 false-positive endoscopic examination findings occurred at the adenoid; in these 6 patients, the MR imaging finding was negative for NPC on the basis of the symmetric striped appearance of the enlarged adenoid (grade 2), indicating benign lymphoid hyperplasia (Fig 4).

Nasopharyngeal MR imaging had a higher sensitivity than endoscopy or endoscopic biopsy for the detection of NPC (100\% versus $88 \%$ and $94 \%$, respectively), the difference being statistically significant between MR imaging and endoscopy $(P=.003)$, and of borderline significance between MR imaging and endoscopic biopsy $(P=.059)$. Nasopharyngeal MR imaging had a lower specificity for the detection of NPC than endoscopy or endoscopic biopsy (92\% versus $94 \%$ and $100 \%$, respectively); the difference was statistically significant between MR imaging and endoscopic biopsy $(P=.002)$, but not between MR imaging and endoscopy $(P=.617)$.

\section{DISCUSSION}

The results from this long-term follow-up study show that MR imaging is a highly sensitive tool for NPC detection because all biopsy-proven NPCs in the initial and 3-year follow-up study had been detected on the initial evaluation by MR imaging. By comparison, tumors in $12 \%$ and $6 \%$ of patients with NPC confirmed at histology were missed by initial endoscopy and by endoscopic biopsy, respectively. In our opinion, these findings demonstrate the necessity of greater use of MR imaging for the diagnostic work-up of patients with suspected NPC because neither endoscopy nor endoscopy plus biopsy can exclude NPC.

A particular strength of MR imaging is that it can assess the nasopharyngeal recess, which is where most NPCs originate. This site is often difficult to inspect endoscopically due to its lateral projection and apposition of its anterior and posterior walls. In 1 patient in the study, both initial and repeat MR imaging examinations detected an NPC within the pharyngeal recess that was missed on initial endoscopy and biopsy and on a repeat biopsy 


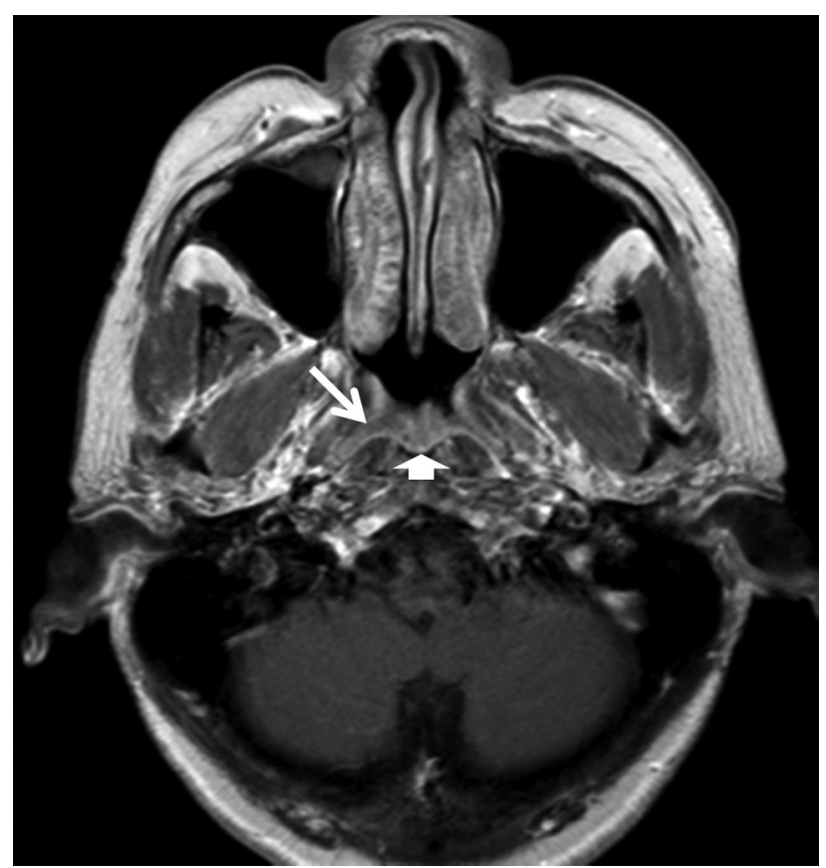

FIG 3. Axial T1-weighted postcontrast MR imaging of a 54-year-old man with lymphoid hyperplasia. MR imaging shows a smooth band with mild enhancement in the right side of the walls of the nasopharynx (arrows), extending from the adenoid (arrowhead), which is asymmetric in thickness compared with the left side and had been misdiagnosed as positive for NPC by MR imaging (grade 3 ).

that was targeted to the MR imaging-detected abnormality, and it was only finally confirmed after a repeat biopsy several years later. In this regard, blind biopsies of this site can be hazardous due to the close proximity of the internal carotid artery to the lateral aspect of the recess; and as in our case, deeply sited small tumors may be missed even with targeted biopsies. These findings highlight the importance of maintaining a high index of suspicion for NPC even if initial biopsies are negative for it.

The optimal management of patients with a suspected but unproved small deeply sited tumor on MR imaging is unclear, though it probably should entail a combination of close imaging surveillance with MR imaging to document whether abnormalities persist or regress, serology, and endoscopy with repeat biopsies as appropriate. In this respect, the optimum timing, frequency, and duration of follow-up MR imaging examinations are unknown. Our example demonstrated only a small increase in tumor size during $>3$ years, suggesting that early NPCs may have slow growth initially. In fact, there is a paucity of data on growth rates of early NPCs, though published reports ${ }^{11}$ of long latency periods between finding an abnormal serology test result and the eventual proof of NPC could be explained by small tumors that remain endoscopically occult for several years. The role of FDGPET in early NPC detection is unclear because although NPCs are typically metabolically active, small tumors may have insufficient metabolic load to be detectable and may be obscured by metabolic activity due to normal lymphatic tissue and/or concomitant inflammatory changes in the nasopharynx. In those regions of the world where NPC is endemic, there are major resource implications for further imaging of these patients; therefore, follow-up is usually based on endoscopy plus or minus serology testing. How-

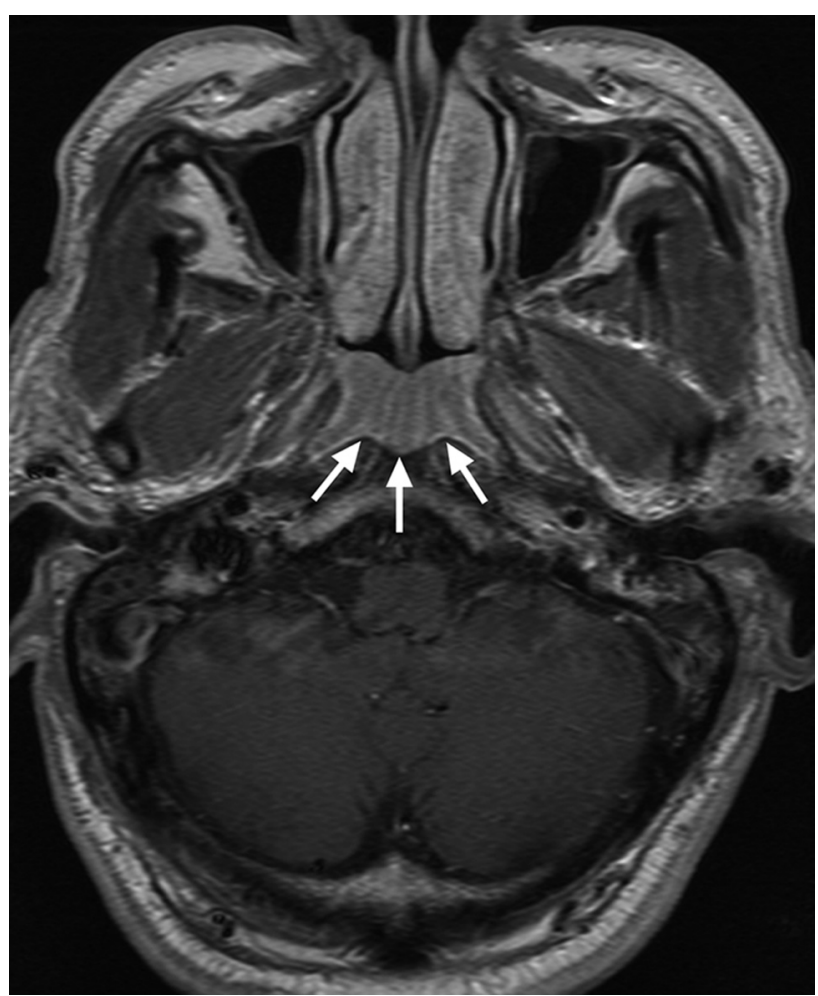

FIG 4. Axial T1-weighted postcontrast MR imaging of a 69-year-old man with benign lymphoid hyperplasia in the adenoid (arrows), which had been positive for NPC by endoscopy but was correctly diagnosed as benign by endoscopic biopsy and MR imaging on the basis of the symmetric alternating bands of marked and mild contrast enhancement causing a striped appearance to the enlarged adenoid (grade 2).

ever, the results of this long-term study suggest that further imaging, by FDG-PET/CT or serial MR imaging, would be best used for those patients with a grade 4 tumor on MR imaging because all of these patients had proved NPC.

The present study confirmed that MR imaging had a 100\% negative predictive value for NPC, which supports the previous conclusion that invasive biopsies are not required when MR imaging findings are negative, especially when the endoscopic examination findings are also negative. ${ }^{10}$ These findings also suggest that a negative MR imaging finding could potentially override a positive endoscopy result, in which a suspicious midline nasopharyngeal mass is seen on endoscopy that is characteristic of adenoidal hyperplasia on MR imaging, based on the presence of a mass with a symmetric striped or striated appearance. ${ }^{12}$

All patients with a tumor identified by MR imaging (grade 4) had NPC, but MR imaging had an imperfect specificity (92\%), which was attributable to false-positive cases in patients with asymmetry in an otherwise generalized lymphoid hyperplasia MR imaging pattern (grade 3). Clearly, it would be beneficial to improve the positive predictive value of MR imaging to avoid unnecessary biopsies in healthy subjects, especially if MR imaging is to be used more widely to support NPC serology screening programs in areas with endemic NPC. In this respect, of those patients with a grade 3 positive MR imaging finding, only those who also had an accompanying positive endoscopy examination finding had NPC. Therefore, we speculate that a grade 3 pattern should undergo biopsy only if the endoscopic examination also 
shows a suspected tumor, though caution would still be advised for those patients with asymmetry in a striped pattern of the adenoid because the internal structure of the adenoid cannot be visualized endoscopically.

The only method that currently can reliably exclude NPC in this patient population is follow-up to ensure that patients did not have a subclinical tumor at initial presentation. Follow-up was performed by re-examination of the nasopharynx at a minimum of 3 years, with a mean of almost 5 years, but 1 remaining limitation of the study is that patients with small slow-growing cancers may not have been identified.

\section{CONCLUSIONS}

MR imaging should be used more widely as a complementary tool to endoscopy and endoscopic biopsy for the detection of nasopharyngeal carcinoma. MR imaging identifies small tumors that cannot be identified through the endoscope, especially those in the pharyngeal recess, where MR imaging may identify a tumor several years before it becomes endoscopically visible. The results of this long-term follow-up study confirm that a normal or symmetric lymphoid hyperplasia pattern in the nasopharyngeal walls or adenoid (grade 1 or 2 ) by MR imaging has a high negative predictive value for NPC. This result supports the assertion that following a normal endoscopy examination finding or one that shows an adenoidal mass, invasive biopsies are not required when MR imaging shows these patterns. Finally, asymmetry in the generalized lymphoid hyperplasia pattern (grade 3) on MR imaging had a low positive predictive value for NPC, and in this study, only those who also had an accompanying positive endoscopy examination finding had NPC.

Disclosures: Ann D. King, Alexander C. Vlantis, Tom Wing Cheung Yuen, Benjamin King Hong Law, Kunwar S. Bhatia, Benny C.Y. Zee, John Woo, Anil T. Ahuja-RELATED: Grant: Research Grants Council of the Hong Kong,* Comments: Project No. CUHK4656/12 and SEG_CUHK02. Anthony T.C. Chan-UNRELATED: theme-based research from the Research Grants Council of Hong Kong*; Other: research funding from Pfizer, * Boehringer Ingelheim, ${ }^{*}$ Merck Serono, ${ }^{*}$ Bristol-Myers Squibb, * Eli Lilly. ${ }^{*}$ K.C. Allen Chan-UNRELATED: Consultancy: I am a consultant to Xcelom Limited; Patents (planned, pending or issued): I have filed patents/patent applications on technologies related to noninvasive diagnostics based on nucleic acid analysis; Roy- alties: The Chinese University of Hong Kong receives royalties on technologies related to noninvasive diagnostic tests based on nucleic acid analysis*; Stock/Stock Options: The Chinese University of Hong Kong holds equities in Sequenom. * Money paid to the institution

\section{REFERENCES}

1. Lee AW, Ng WT, Chan LL, et al. Evolution of treatment for nasopharyngeal cancer: success and setback in the intensity-modulated radiotherapy era. Radiother Oncol 2014;110:377-84 CrossRef Medline

2. Chan KC, Hung EC, Woo JK, et al. Early detection of nasopharyngeal carcinoma by plasma Epstein-Barr virus DNA analysis in a surveillance program. Cancer 2013;119:1838-44 CrossRef Medline

3. Wen YH, Zhu XL, Lei WB, et al. Narrow-band imaging: a novel screening tool for early nasopharyngeal carcinoma. Arch Otolaryngol Head Neck Surg 2012;138:183-88 CrossRef Medline

4. Wang WH, Lin YC, Lee KF, et al. Nasopharyngeal carcinoma detected by narrow-band imaging endoscopy. Oral Oncol 2011;47: 736-41 CrossRef Medline

5. Gao Y, Zhu SY, Dai Y, et al. Diagnostic accuracy of sonography versus magnetic resonance imaging for primary nasopharyngeal carcinoma. J Ultrasound Med 2014;33:827-34 CrossRef Medline

6. Olmi P, Fallai C, Colagrande S, et al. Staging and follow-up of nasopharyngeal carcinoma: magnetic resonance imaging versus computerized tomography. Int J Radiat Oncol Biol Phys 1995;32:795-800 CrossRef Medline

7. Chong VF, Fan YF, Khoo JB. Nasopharyngeal carcinoma with intracranial spread: CT and MR characteristics. J Comput Assist Tomogr 1996;20:563-69 CrossRef Medline

8. King AD, Lam WW, Leung SF, et al. MR imaging of local disease in nasopharyngeal carcinoma: tumour extent vs tumour stage. $\mathrm{Br} J$ Radiol 1999;72:734-41 CrossRef Medline

9. King AD, Vlantis AC, Tsang RK, et al. Magnetic resonance imaging for the detection of nasopharyngeal carcinoma. AJNR Am J Neuroradiol 2006;27:1288-91 Medline

10. King AD, Vlantis AC, Bhatia KS, et al. Primary nasopharyngeal carcinoma: diagnostic accuracy of MR imaging versus that of endoscopy and endoscopic biopsy. Radiology 2011;258:531-37 CrossRef Medline

11. Ji MF, Wang DK, Yu YL, et al. Sustained elevation of Epstein-Barr virus antibody levels preceding clinical onset of nasopharyngeal carcinoma. Br J Cancer 2007;96:623-30 CrossRef Medline

12. Bhatia SS, King AD, Vlantis AC, et al. Nasopharyngeal mucosa and adenoids: appearance at MR imaging. Radiology 2012;263:437-43 CrossRef Medline 